\title{
Synchronization Phenomena in Coupled Logistic Maps Involving Parametric Force
}

\author{
Hironori Kumeno \\ Dept. of Electrical and Electronic Eng., \\ Tokushima University, \\ Tokushima, 770-8506 JAPAN \\ Email: kumeno@ee.tokushima-u.ac.jp
}

\author{
Yoshifumi Nishio \\ Dept. of Electrical and Electronic Eng., \\ Tokushima University, \\ Tokushima, 770-8506 JAPAN \\ Email: nishio@ee.tokushima-u.ac.jp
}

\begin{abstract}
Synchronization phenomena in coupled logistic maps whose parameters are forced into periodic varying are investigated through the use of Lyapunov exponents. When three maps are coupled, various synchronization phenomena are observed by choosing a coupling intensity. The synchronization phenomena fall into three general categories, which are asynchronous, synchronization of two among the three maps and synchronization of all the maps. In particular, in the synchronization of two of the three maps, solutions of maps behave periodic, quasi-periodic and chaotic for several coupling intensities.
\end{abstract}

\section{INTRODUCTION}

Synchronization is one of the fundamental phenomena in nature, and one of typical nonlinear phenomena. Therefore, studies on synchronization phenomena of coupled systems are extensively carried out in various fields, physics [1], biology [2], engineering and so on. Parametric excitation circuit is one of resonant circuits, and it is important to investigate various nonlinear phenomena for future engineering applications. In a simple oscillator including parametric excitation, Ref. [3] reports that the almost periodic oscillation occurs if nonlinear inductor has saturation characteristic. Additionally the occurrence of chaos is referred in Refs. [4] and [5]. The network of chaotic elements can be modeled by a system of coupled one-dimensional maps. Behavior generated in coupled system of chaotic one-dimentional map is investigated in Refs. [6][8] In particular, Coupled Map Lattice (CML) and Globally Coupled Map (GCM) are well known as mathematical models in discrete-time system. The research into CML and GCM is important for not only modeling of multiple degree of freedom nonlinear systems but also application to biological networks and engineering. In the past we have investigated effects of parametric excitation of coupled van der Pol oscillators [9]. In this study, for more detailed investigation of the effect of parametric excitation on synchronization, we focus on a globally coupled system of simple one-dimensional maps. A typical scheme for global coupling is given by

$$
\begin{aligned}
& x_{i}(t+1)=(1-\varepsilon) f\left[x_{i}(t)\right]+\frac{\varepsilon}{N} \sum_{j=1}^{N} f\left[x_{j}(t)\right] \\
& i=1,2, \cdots, N
\end{aligned}
$$

where $\varepsilon \in[0,1]$ is the coupling intensity. The globally coupled maps are a scheme that an average number of all the maps affect each of the map, and similar to the perfect coupling system of oscillators that we have studied using van der Pol oscillators. The one-dimensional map used in this study is a logistic map, since the map can be described by a simple discrete equation. Mathematically, the logistic map is written as

$$
x(t+1)=\alpha x(t)(1-x(t)) .
$$

In this study, we investigate synchronization phenomena in the coupled logistic maps whose parameters are forced into periodic varying. When three maps are coupled, various synchronization phenomena are observed by choosing a coupling intensity. The synchronization phenomena fall into three general categories, which are asynchronous, synchronization of two of the three maps and synchronization of all the maps. In three state space corresponding to the three coupled map, the synchronization phenomena are investigated through spread of solutions, and classified according to behavior of the solutions. Thus, Lyapunov exponent that calculate the temporal spreading of the solutions is available for investigate the synchronization. The synchronization is investigated quantitatively through comparing three-dimensional Lyapunov exponent with the synchronization.

\section{Parametrically forced logistic MaP}

A parametrically forced logistic map used in this study is described as:

$$
x(t+1)=\alpha_{f}(t) x(t)(1-x(t)),
$$

and

$$
\alpha_{f}(t)=\left\{\begin{array}{cc}
\alpha_{1}, & n\left(\tau_{1}+\tau_{2}\right)<t \leq n\left(\tau_{1}+\tau_{2}\right)+\tau_{1} \\
\alpha_{2}, & n\left(\tau_{1}+\tau_{2}\right)+\tau_{1}<t \leq(n+1)\left(\tau_{1}+\tau_{2}\right) \\
(n=1,2, \ldots)
\end{array},\right.
$$

where $\alpha_{f}(t)$ is a term of the parametric force and timevarying. The parametric force operation can be described as follows: in the time interval $n\left(\tau_{1}+\tau_{2}\right)<t \leq n\left(\tau_{1}+\tau_{2}\right)+\tau_{1}$, the system is driven by parameter $\alpha_{1}$ during the duration $\tau_{1}$; while in the interval $n\left(\tau_{1}+\tau_{2}\right)+\tau_{1}<t \leq(n+1)\left(\tau_{1}+\tau_{2}\right)$, the system is driven by parameter $\alpha_{2}$ during the duration $\tau_{2}$. In this system, two maps which have two kinds of parameters are replaced alternately by the number of updates. Then, a 

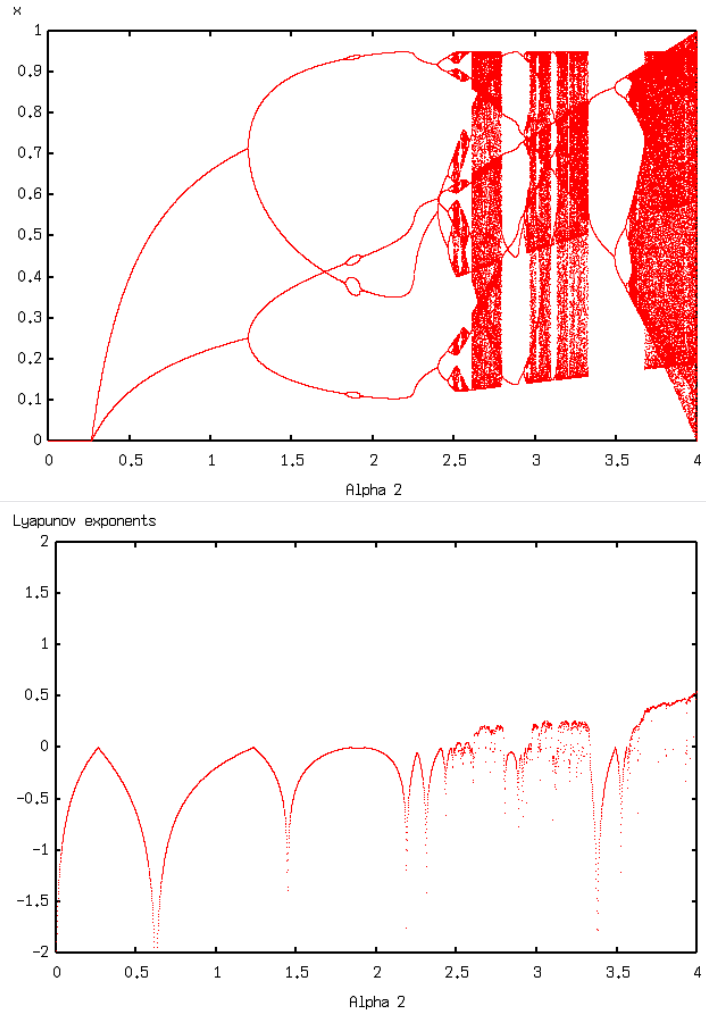

Fig. 1. One-parameter bifurcation diagram (top) and the Lyapunov exponents (bottom) for $\alpha_{1}=3.8$ and $\tau=1$. Horizontal axis: $\alpha_{2}$.

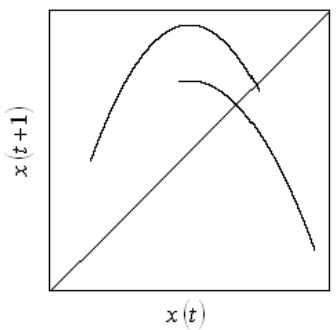

(a)

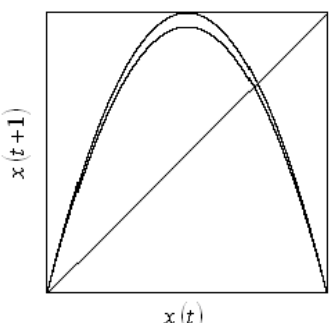

(b)
Fig. 2. Return maps of parametrically forced logistic maps for $\tau=1$. (a) $\alpha_{1}=3.0$ and $\alpha_{2}=3.83$. (b) $\alpha_{1}=3.8$ and $\alpha_{2}=4.0$.

parameter giving a periodic solution and a parameter giving another periodic solution can be combined. Of course, other combinations, for instance two parameters giving a periodic solution and chaotic solution or two parameters giving two kinds of chaotic solutions, are possible. In this study, we assume $\tau_{1}=\tau_{2}=\tau$ for simplicity.

The one-parameter bifurcation diagrams and the Lyapunov exponents are obtained as shown in Fig. 1. From the figures, observations of periodic and chaotic attractors are confirmed. Figure 2 show some examples of the return maps of the parametrically forced logistic maps. For the original logistic map, two-periodic solution is observed for $\alpha=3.0$. While, three-periodic solution is observed for $\alpha=3.83$. These two solutions are periodic, whereas in the logistic map involving parametric force, a solution is chaotic as shown in Fig. 2(a) when the parameters $\alpha_{1}$ and $\alpha_{2}$ are set 3.0 and 3.83. Namely, chaotic solution can be observed in the combination of two parameters that generate two kinds of periodic solutions.

\section{SYNCHRONIZATION}

Synchronization phenomena generated in the coupled logistic map involving parametric force are investigated for one control parameter $\varepsilon$ which is coupling intensity when three maps are coupled and $\tau=1$. Given that state spases of the three maps are a three-dimensional phase spase, threedimensional Lyapunov exponent is defined because there exist Lyapunov exponent whose the number of dimension is the same as the number of dimension of the phase spase. The three-dimensional Lyapunov exponents obtained at $\alpha_{1}=3.8$, $\alpha_{2}=4.0$ and $\tau=1$ are shown in Fig. 3. In Fig. 3, $\lambda_{1}$ is maximal Lyapunov exponent. $\lambda_{2}$ is a Lyapunov exponent whose direction is orthogonal to that of the $\lambda_{1} \cdot \lambda_{3}$ is a Lyapunov exponent whose direction is orthogonal to that of the $\lambda_{1}$ and that of the $\lambda_{2}$. Thus, $\lambda_{1}$ calculates how complex trafection of the solution is. $\lambda_{1}+\lambda_{2}$ calculates planar spread of the solutions. $\lambda_{1}+\lambda_{2}+\lambda_{2}$ calculates spatial spread of the solutions. Figure 4 shows attractors in three-dimensional phase space. Spatial spreads of the solutions are investigated by using Fig. 3(b). In Fig. 3(b), as increasing $\varepsilon$, for $\varepsilon>0.19$, $\lambda_{1}+\lambda_{2}+\lambda_{3}$ becomes negative, whereas $\lambda_{1}+\lambda_{2}$ is positive. Namely, the solutions narrow spatially, whereas the solutions spread planarly. Though, more increasing $\varepsilon, \lambda_{1}+\lambda_{2}$ suddenly become negative. The solutions narrow planarly. However, for $\varepsilon \geq 0.32, \varepsilon>0.19, \lambda_{1}+\lambda_{2}+\lambda_{3}$ become positive again.

Figure 5 shows observed synchronization phenomena corresponding to Fig. 4. In Fig. 5, upper figures show the return maps and lower figures show the phase differences between the maps. For small value of the coupling intensity $\varepsilon, \lambda_{1}$ is positive and $\lambda_{2}$ and $\lambda_{3}$ is almost the same as $\lambda_{1}$. All the maps are chaotic and not synchronized as shown in Fig. 5(a). For $\varepsilon \geq 0.17, \lambda_{3}$ is close to $\lambda_{2}$, two of the three maps are synchronized as shown in Fig. 5(b). In the figure, $x_{1}$ equal to $x_{2}$. Thus, map 1 and map 2 are synchronized. However, in relation of phase between maps 2 and map 3, concentration distribution of the phase is not uniform state, and two areas are high density. Thus, it seem that some kind of relation of phase exists beteen the unsynchronized maps. For $\varepsilon>0.18$, $\lambda_{3}$ becomes negative. All chaotic solutions gather in two areas as shown in Fig. 5(c). In the figures of the phase differences in Fig. 5(c), the areas where the solutions exist are symmetrical about the axis of $x_{2}(t)=x_{3}(t)$. That means, if $x_{2}$ is in an area around $x_{2}=0.5, x_{3}$ is in an area around $x_{3}=0.8$. While, if $x_{2}$ is in the area around $x_{2}=0.8, x_{3}$ is in the area around $x_{3}=0.5$. For $\varepsilon>0.19, \lambda_{2}$ becomes negative. In Fig. 5(d), the phase difference between maps which are not synchronized become like circle. For $\varepsilon>0.22, \lambda_{1}$ becomes zero. That means the solutions of all the maps are quasi-periodic. For $\varepsilon>0.24, \lambda_{1}$ becomes negative. That means the solutions of all the maps are periodic (see Fig. 5(e)). For $\varepsilon>0.32, \lambda_{1}$, $\lambda_{2}$ and $\lambda_{3}$ becomes positive. All the maps become chaotic as shown in Fig. 5(f). Additionally, two of the three maps 


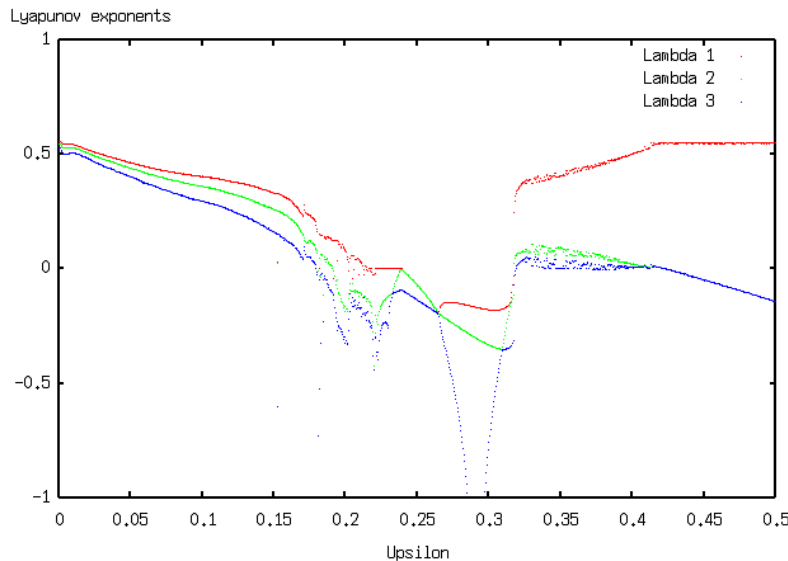

(a)

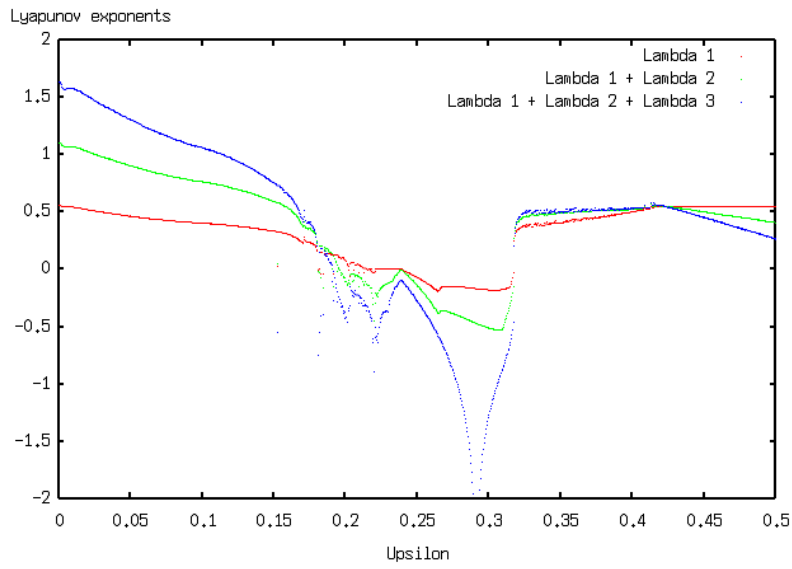

(b)

Fig. 3. Lyapunov exponents in globally coupled parametrically forced logistic maps for $\alpha_{1}=3.8, \alpha_{2}=4.0$ and $\tau=1$. Horizontal axis: $\varepsilon$. (a)Each of $\lambda$. (b)Sums of $\lambda$.

remain in the synchronization. In the figure, the solutions in all the maps spread over solution space. Though, the shapes of the return maps are different from that of the uncoupled map which are introduced in Fig. 2(b). For $\varepsilon>0.42, \lambda_{2}$ and $\lambda_{3}$ becomes the same. All the map are synchronized as shown in Fig. 5(g). In the figure, all the maps are chaotic and have the same shapes as a map of the uncoupled map.

\section{CONCLUSION}

In this study, we investigated synchronization phenomena in the coupled logistic maps which are forced into periodic parameter varying. When three maps are coupled, various synchronization phenomena are observed by choosing the coupling intensity. The synchronization phenomena fall into three general categories, which are asynchronous, synchronization of two among the three maps and synchronization of all the maps. In particular, in the synchronization of two of the three maps, solutions of maps behave periodic, quasiperiodic and chaotic for several coupling intensities. Moreover, the synchronization was investigated quantitatively through comparing three-dimensional Lyapunov exponent with the synchronization.
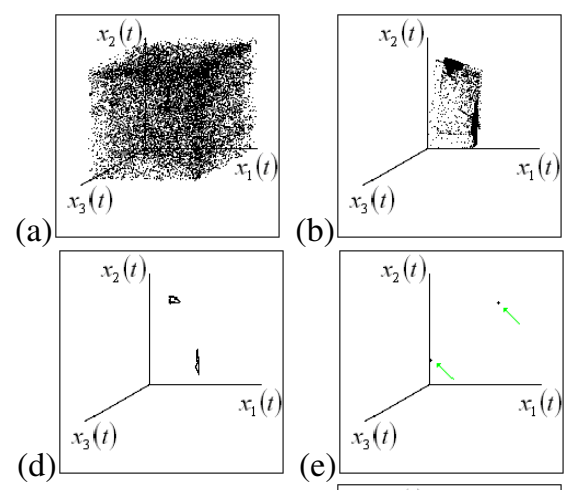

(b)

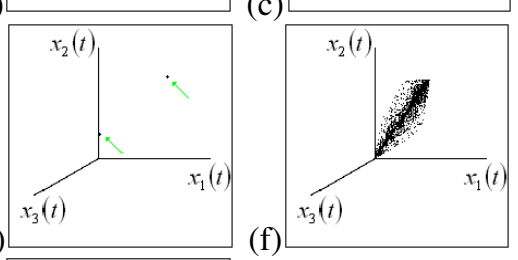

(g)
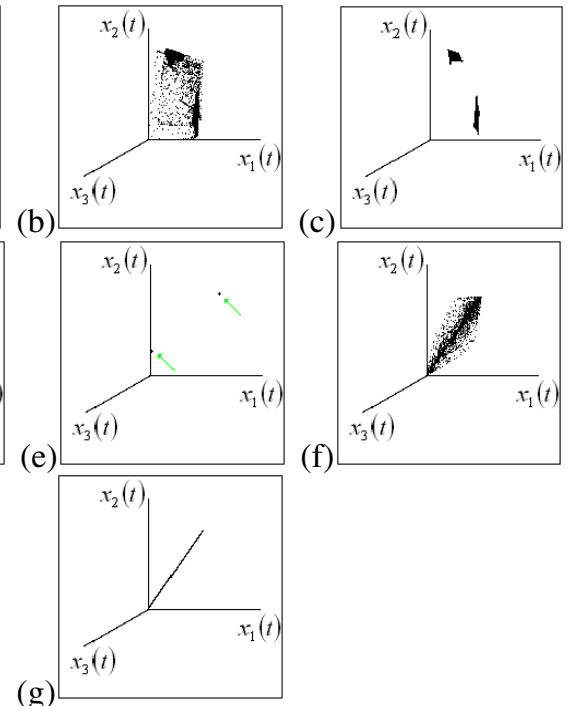

(c)

Fig. 4. Attractors in three-dimensional phase space for $\alpha_{1}=3.8, \alpha_{2}=4.0$ and $\tau=1$. (a) $\varepsilon=0.100$. (b) $\varepsilon=0.180$. (c) $\varepsilon=0.185$. (d) $\varepsilon=0.205$. (e) $\varepsilon=0.300$. (f) $\varepsilon=0.320$. (g) $\varepsilon=0.410$.

\section{ACKNOWLEDGMENT}

This work was partly supported by Yazaki Memorial Foundation for Science and Technology.

\section{REFERENCES}

[1] I. Belykh, M. Hasler, M. Lauret and H. Nijmeijer, "Synchronization and graph topology," Int. J. Bifurcation and Chaos, vol.15, no.11, pp.34233433, Nov. 2005

[2] J. Cosp, J. Madrenas, E. Alarcon, E. Vidal and G. Villar, "Synchronization of nonlinear electronic oscillators for neural computation," IEEE Trans. Neural Networks, vol.15, no.5, pp.1315-1327, Sep. 2004.

[3] C. Hayashi, "Nonlinear Oscillations in Physical Systems," Chap. 11, McGraw-Hill, New York (1964).

[4] C. Hayashi, M. Abe, K. Oshima and H. Kawakami, "The Method of Mapping As Applied To the Solution for Certain Types of Nonlinear Differential Equations," Proc. of the Ninth International Conference on Nonlinear Oscillations, pp. 1-8, 1981.

[5] M. Inoue, "A Method of Analysis for the Bifurcation of the Almost Periodic Oscillation and the Generation of Chaos in a Parametric Excitation Circuit," Trans. of IEICE, vol. J68-A, no. 7, pp. 621-626, 1985.

[6] K. Kaneko, "Spatiotemporal intermittency in couppled map lattice," Prog. Theor. Phys., vol.74, no.5, pp.1033-1044, Nov. 1985.

[7] K. Kaneko, "Pattern dynamics in spatiotemporal chaos," Physica D, vol.34, nos.1-2, pp.1-41, Jan. 1989.

[8] K. Kaneko, "Clustering, coding, switching, hierarchical ordering, and control in a network of chaotic elements," Physica D, vol.41, nos.2, pp.137-172, March. 1990.

[9] H. Kumeno and Y. Nishio, "Synchronization Phenomena in Coupled Parametrically Excited van der Pol Oscillators," Proceedings of International Symposium on Nonlinear Theory and its Applications (NOLTA' 08), pp. 128- 131, Sep. 2008 


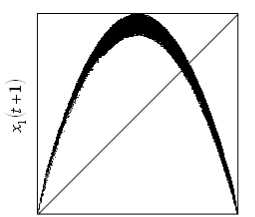

$x_{1}(t)$

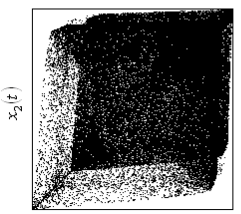

(a)
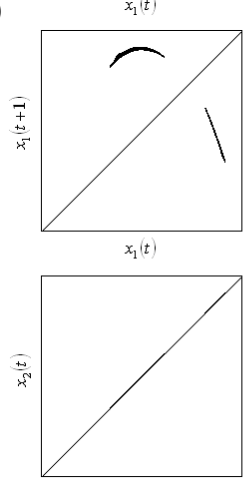

(c)

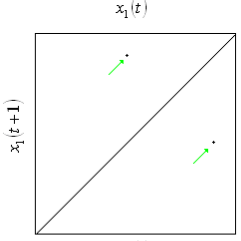

$x_{1}(t)$

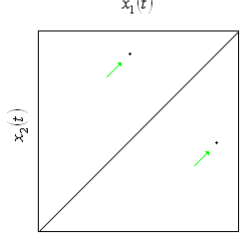

(e)

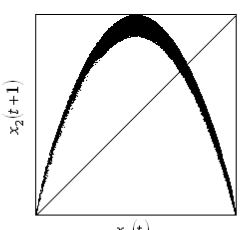

$x_{2}(t)$
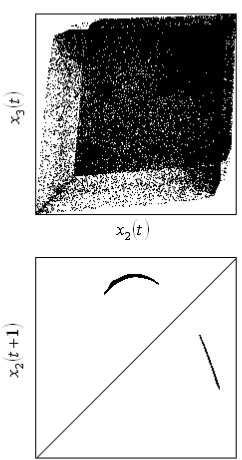

$x_{2}(t)$
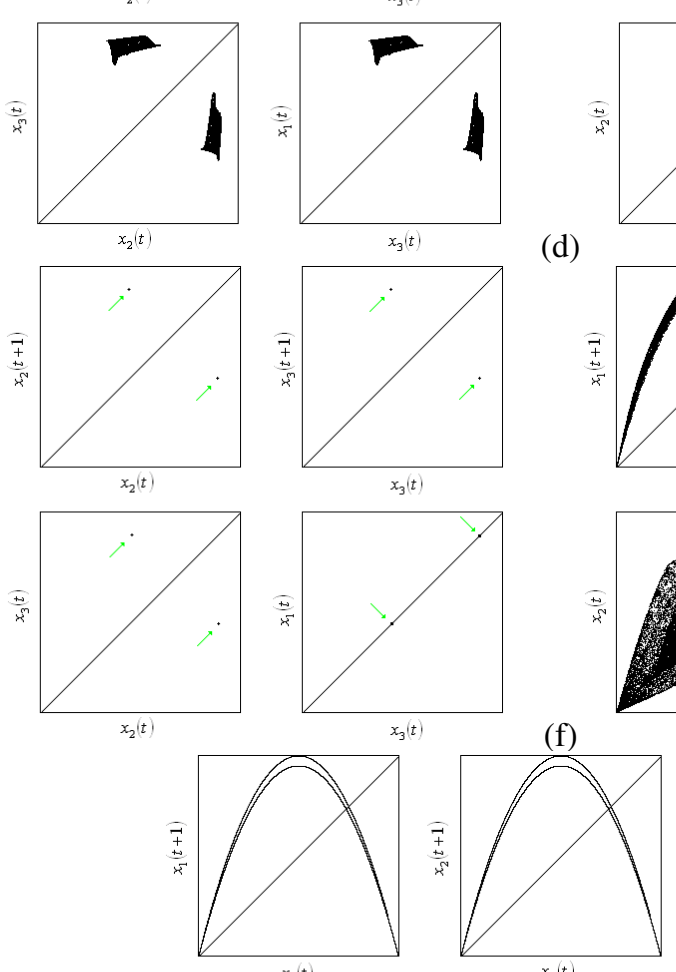

(d)

(b)
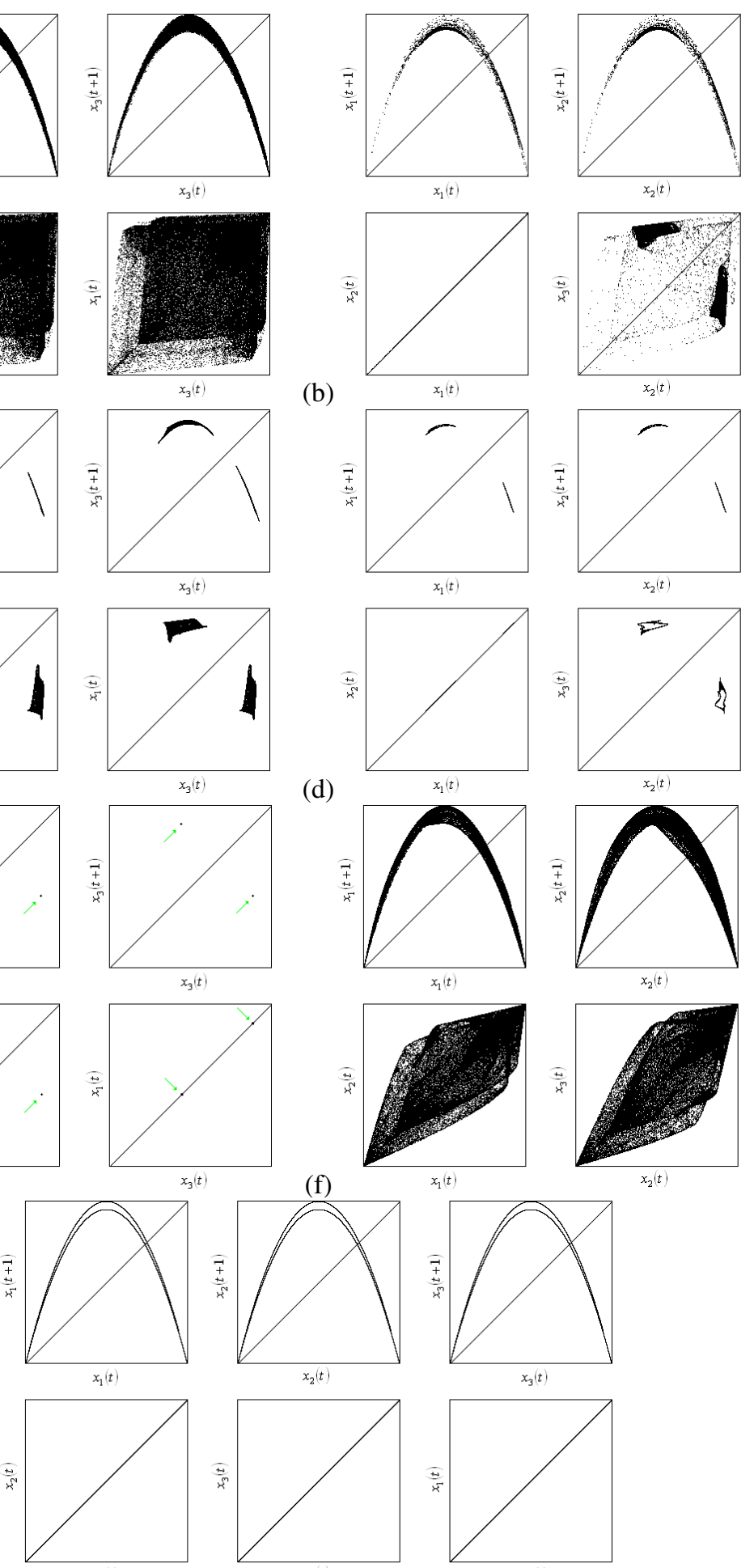

(g)
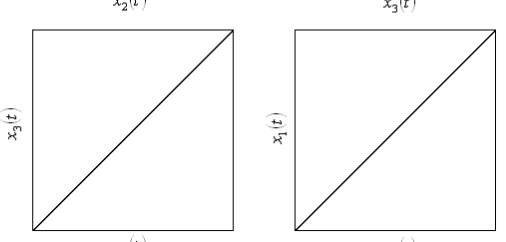
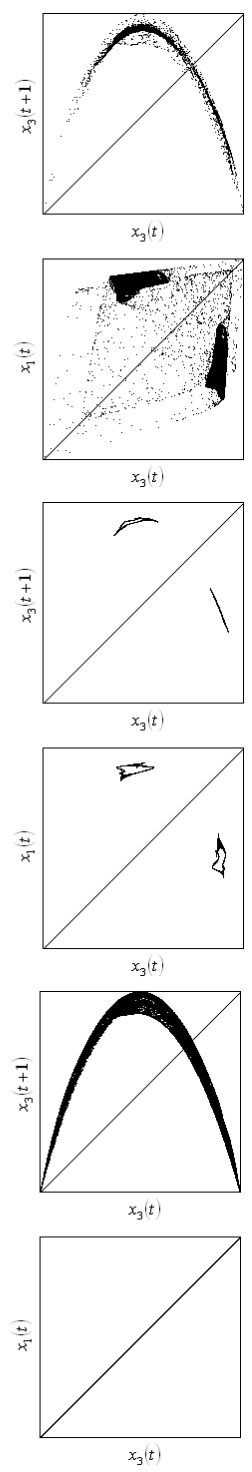

Fig. 5. Return maps and phase differences for $\alpha_{1}=3.8, \alpha_{2}=4.0$ and $\tau=1$. (a) $\varepsilon=0.100$. (b) $\varepsilon=0.180$. (c) $\varepsilon=0.185$. (d) $\varepsilon=0.205$. (e) $\varepsilon=0.300$. (f) $\varepsilon=0.320$. (g) $\varepsilon=0.410$. 